\title{
The Application of Mesoturbulence to Stellar Atmospheres
}

\author{
E. Sedlmayr \\ Institut für Theoretische Astrophysik \\ der Universität Heidelberg \\ Heidelberg, FRG
}

\section{Abstract}

For realistic stellar atmospheres the equations describing mesoturbulent line formation are solved numerically. The general dependence of theoretical line profiles and equivalent widths on the correlation length $I$ and the mean square turbulent velocity $\sigma$ is demonstrated. Also empirical relations between the basic parameters of the micro-macroturbulence description ( $v_{\text {mic }}, v_{\text {mac }}$ ) and the fundamental mesoturbulence parameters $(1, \sigma)$ are derived.

\section{Introduction}

It has been shown (see Traving's contribution) that turbulence in stellar atmospheres is necessarily of finite scale length. So mesoturbulence is a very common phenomenon which should be taken into account in line formation studies.

In this contribution we shall apply the mesoturbulence formalism (e.g. Auvergne et al. (1973), Gail et al. (1974), Gail and Sedlmayr (1974), Frisch (1975)) to line formation in realistic atmospheres. our aimes are

(i) to demonstrate the basic effects of the correlation length 1 and the mean square turbulent velocity $\sigma$ on the line profiles and equivalent widths, and

(ii) to derive empirical relations between the classical parameters (microturbulent velocity $v_{m i c}$ and macroturbulent velocity $v_{\text {mac }}$ ' and the fundamental parameters of the mesoturbulence description 1 and $\sigma$. 


\section{The Numerical Method}

Two particular stochastic models have been adopted for discussing mesoturbulent line formation in stellar atmospheres:

a) The Uhlenbeck-Ornstein Process (UOP)

The UOP is a stationary Markovian process continuous in space with both Gaussian one-point and two-point velocity distribution functions. The resulting transfer equation for the monochromatic local conditional intensity $q(s, v)$ is therefore a parabolic partial differential equation of Fokker-Planck type (Gail et al. (1974), Gail and Sedlmayr (1974):

$$
\frac{\partial q}{\partial s}=\frac{1}{I}\left(-v \frac{\partial g}{\partial v}+\sigma^{2} \frac{\partial^{2} q}{\partial v^{2}}\right)-\left(k_{0} \phi+k_{c}\right)(q-s),
$$

with $s$ being the distance along the ray, $v$ the actual velocity, $\kappa_{0} \phi$ and $k_{c}$ the line and continuum absorption coefficients. $\phi(s, v, \sigma, \Delta \lambda)$ is the profile function and $s$ the monochromatic local source function. $\kappa_{o}, \kappa_{c}$ and $s$ are assumed to be independent of the velocity $v$.

The expectation value for the monochromatic local intensity $\langle I$ (s) > is obtained by multiplication of $\mathrm{q}$ by the one-point velocity distribution $\mathbb{P}_{1}(v)$ and integration with respect to the velocity variable:

$$
\langle\mathrm{I}(\mathrm{s})\rangle=\int_{-\infty}^{+\infty} \mathrm{dv} \mathbf{P}_{1}(\mathrm{v}) \mathrm{q}(\mathrm{s}, \mathrm{v})
$$

Given a model atmosphere which provides $\kappa_{0}, \kappa_{c}$ and $s$, and given appropriate initial and boundary conditions the above transfer equation can be solved numerically straight-forward by the CrankNicholson method (e.g. Richtmyer and Morton,(1967)).

Without going into any technical details I shall point to the only problem which may arise and which results from an inconsistent incorporation of the boundary conditions: The natural boundary conditions for our problem are given by the asymptotic limits $\mathrm{v} \rightarrow \pm \infty$, where the line absorption vanishes and the radiative transfer is controlled by continuous absorption only. It turns out that in the case of strong lines or large turbulent velocities one has to take into account rather large $|v|$ to approach these limits with sufficient accuracy and to avoid slight instabilities near the boundaries. 
b) The Kubo-Anderson Process (KAP)

The KAP is a stationary Markovian process discontinuous in space with a Gaussian one-point velocity distribution function (like UOP) but a non-Gaussian two-point velocity distribution function which bridges the gap between the two limiting cases of complete correlation and complete noncorrelation by a linear ansatz (Auvergne et al. (1973), Frisch and Frisch (1975); see also Traving's contribution).

Using the KAP one arrives at an integro-differential equation for the monochromatic local conditional intensity $\mathrm{q}(\mathrm{s}, \mathrm{v})$ :

$$
\frac{\partial q(s, v)}{\partial s}=\frac{1}{I}\left\{f_{-\infty}^{+\infty} d v^{\prime} \mathbb{P}_{1}\left(v^{\prime}\right) q\left(s, v^{\prime}\right)-q(s, v)\right\}-\left(k_{0} \phi+k_{c}\right)(q(s, v)-s) \text {. }
$$

From this equation the expectation value of the emergent intensity can be calculated

(i) by means of the semianalytical methods used by Auvergne et al. (1973), and Frisch (1975) or

(ii) by a direct numerical solution of (3) and a subsequent integration according to (2). A proper discretisation in the velocity coordinate reduces this equation to a system of coupled linear ordinary differential equations which can be solved by standard methods (Gail et al. (1976).

However, both procedures have inherent specific difficulties either connected with instabilities in the region of very small 1 (microturbulent limit) or problems of large matrix size respectively.

Thus for numerical reasons we consider the straigthforward solution of the Fokker-Planck equation described in a) to be more efficient.

2. Effect of a Correlated Velocity Field on Line Profiles and Equivalent Widths

Adopting the UOP line profiles, equivalent widths and curves of growth have been calculated for selected lines of particular elements formed in the solar photosphere. In order to account for the effects of model atmospheres different from the sun we have extended these computations to atmospheres of earlier and later spectral type (AOV (Wega) and K2III (Arcturus)) also. 
All calculated profiles show a similar monotonic dependence on 1 and $\sigma$ respectively. Thus for demonstration of principle effects we may restrict ourselves to the discussion of an arbitrary line in the solar photosphere.

For a given mean square turbulent velocity $\sigma$ and a non-negative correlation function microturbulence always yields the maximum absorption for a spectral line. Hence line profiles which originate in a velocity field determined by a small scale length are always deeper than the corresponding profiles formed in a velocity field with equal turbulent velocity but larger correlation length.

This is clearly seen in Fig. 1a, where for the line FeI $\lambda 6200$ theoretical profiles are plotted for $\sigma=2 \mathrm{~km} / \mathrm{s}$ and different values for the correlation length $1.1=1 \mathrm{~km}$ and $1=10^{4} \mathrm{~km}$ refer to the microturbulent and macroturbulent limit respectively.

If the correlation of the velocity field is increased for fixed $\sigma$ both the line depth and the width of the corresponding profiles decrease monotonically until for very large $1(23000 \mathrm{~km})$ saturation is achieved (macroturbulent limit).

This general behaviour is also demonstrated in Fig. 2 which for different values of $\sigma$ - but for the same line - shows the effect of the correlation length 1 on the central depth $r_{0}$ and the equivalent width $w_{\lambda}$ of the emergent profile.

For $\sigma \lesssim 1 \mathrm{~km} / \mathrm{s}$ thermal broadening exerts the essential influence on the absorption coefficient. Hence for small mean square turbulent velocities both the central depths and equivalent widths depend only slightly on 1 . With increasing $\sigma$ the thermal contribution becomes more and more negligible and the influence of the correlation of the turbulence field on $r_{0}$ and $w_{\lambda}$ is considerably increased.

For large $\sigma$ both $r_{0}$ and $w_{\lambda}$ become monotonically strongly decreasing functions with increasing 1 approaching their minimum value in the macroturbulent limit. In this case the equivalent widths become independent on $\sigma$; a fact which causes all $\mathrm{w}_{\lambda}$ - curves in Fig. 2 to converge asymptotically.

The theory of mesoturbulent line formation provides a simple criterion which allows a quantitative estimate of a profile's deviation from the corresponding microturbulent result (see Traving's contribution). According to this criterion microturbulence should be a good approximation if 
Fig. 1: All calculations have been performed on the basis of the empirical solar model atmosphere of Holweger (1967) assuming a mean square turbulent velocity $\sigma=2 \mathrm{~km} / \mathrm{s}$ and a normal iron abundance.

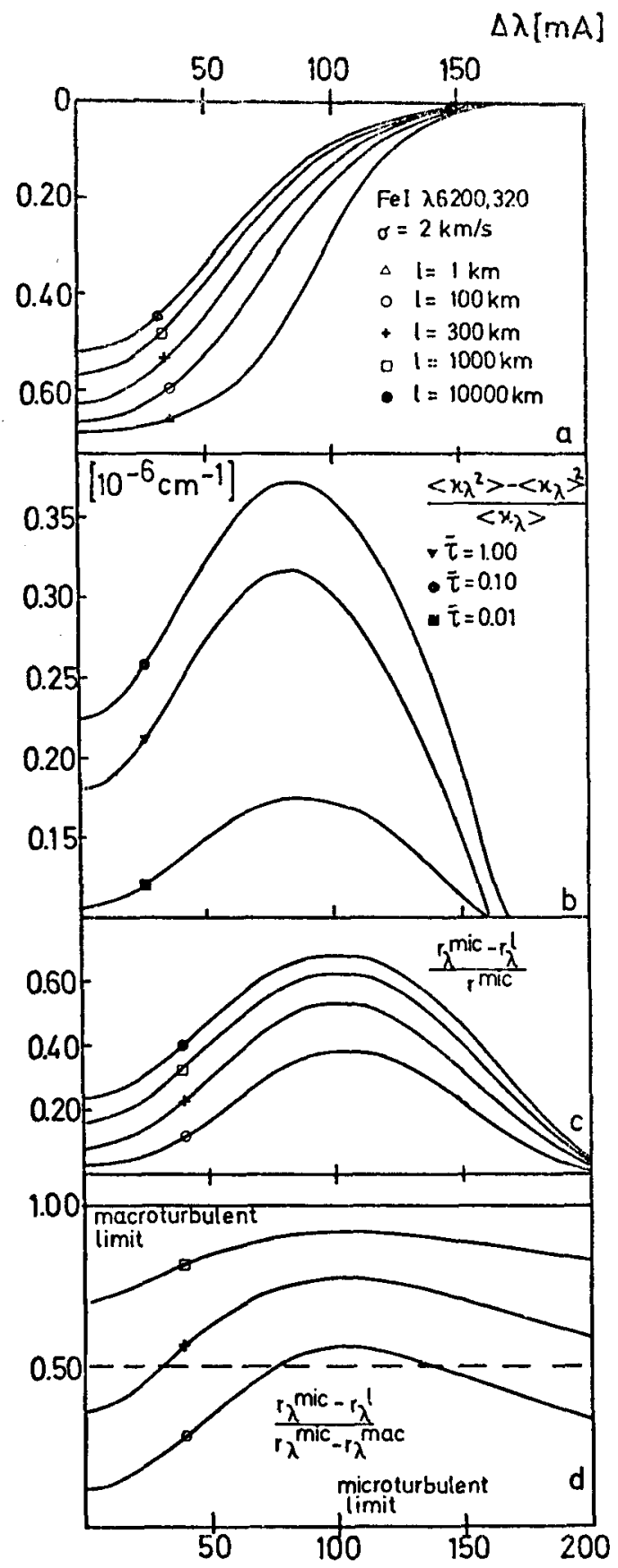

a) Theoretical profiles of the line FeI $\lambda 6200$ for different values of the correlation length $1.1 \lesssim 1 \mathrm{~km}$ and $1 \gtrsim 3000 \mathrm{~km}$ correspond to the micro- and macroturbulent limit respectively.

b) Mean square root of the line absorption coefficient $k_{\lambda}=k(s) \phi(s, v, \sigma, \Delta \lambda)$ versus $\lambda^{\lambda}$ at different optical depths (Rosseland depth scale).

c) Deviation of a profile (calculated with a finite correlation length) from the microturbulent profile normated to the microturbulent value.

d) Deviation of a profile (calculated with a finite correlation length) from the microturbulent profile normated to the difference between the microand macroturbulent profile. This quantity indicates quantitatively which part of a profile is mainly determined by microturbulent or macroturbulent conditions respectively. 


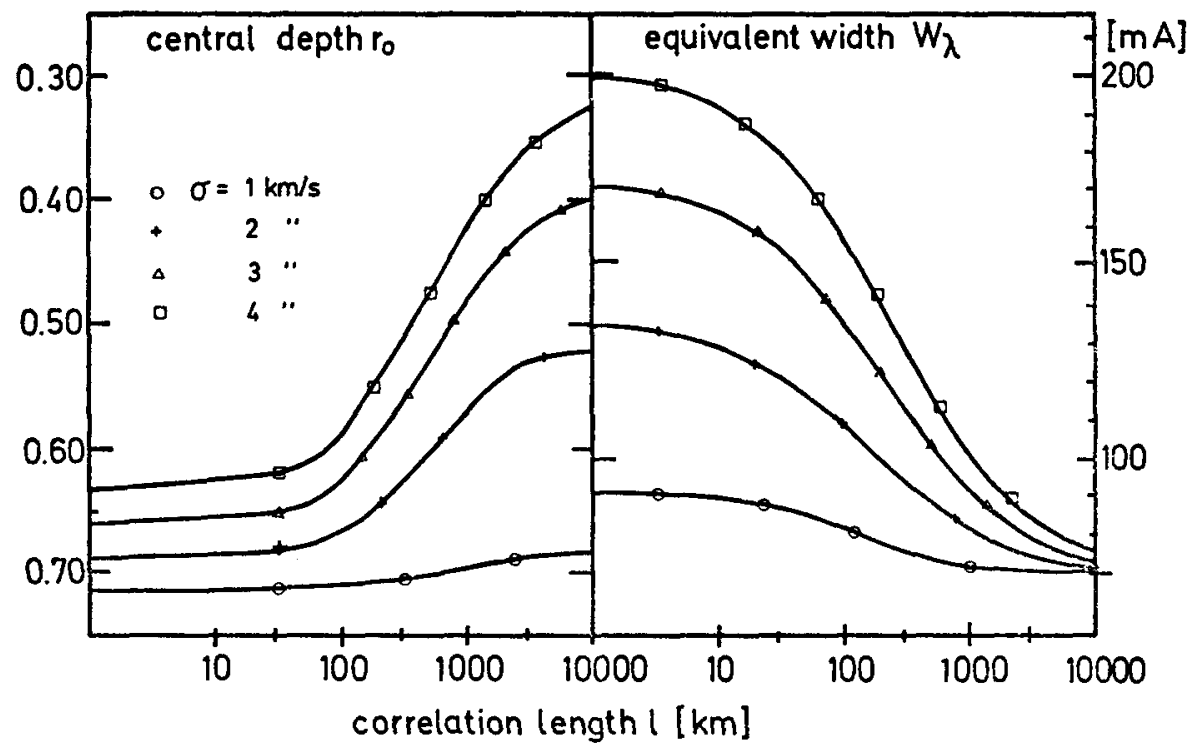

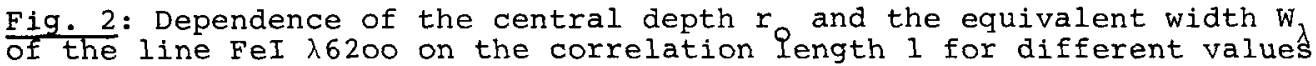
of the mean square turbulent velocity $\sigma$.

the condition

$$
\frac{1}{2} \tau_{I} \cdot \tau_{s} \frac{\left\langle k^{2}\right\rangle-\langle k\rangle^{2}}{\langle k\rangle^{2}}<<1
$$

holds. $\langle\ldots\rangle$ indicates the average values calculated by means of the one-point velocity distribution function, and $\tau_{1}=1\langle k\rangle$ and $\tau_{S}=s\langle k\rangle$ are characteristic optical depths corresponding to the correlation length and the relevant geometrical depth of line formation respectively. Thus by definition $\tau_{s}$ is usually of the order of unity. With $\tau_{s}=1$ and $\tau_{1}=1\langle k\rangle$ the proper wavelength dependence of (4) is essentially given by the quantity $\left(\left\langle k^{2}\right\rangle-\langle k\rangle^{2}\right) /\langle k\rangle$, which is plotted in Fig. 1b for relevant optical depths $\bar{\tau}$.

From Fig. 1b we expect the largest deviations for a line profile calculated with fixed values $1, \sigma$ from the corresponding microturbulent result to occur at the transition from the core to the wing of the line where the curves $\left(\left\langle\kappa^{2}\right\rangle-\langle k\rangle^{2}\right) /\langle k\rangle$ show a pronounced maximum.

However, condition (4) is primarily a mathematical condition which for each wavelength measures the magnitude of the difference between a profile calculated with finite correlation length and the same profile 
calculated under the assumption of microturbulence. Obviously Fig. 1b shows that for the line centre and the outer wings the deviations from the microturbulent profile are considerably smaller than for the transition region even for large 1 . This is confirmed by the results in Fig. 1c where for different values of 1 the normated deviations from the microturbulent profile are plotted versus $\lambda$. However, this does not indicate that for large 1 these parts of the line do form under microturbulent conditions, but only that for these parts of the profile the microturbulent result is a reliable approximation for the true profile.

In order to decide whether micro- or macroturbulent conditions prevail we have plotted in Fig. 1d the quantity

$$
a_{\lambda}^{l, \sigma}=\left(r_{\lambda}^{\text {mic, } \sigma}-r_{\lambda}^{l, \sigma}\right) /\left(r_{\lambda}^{\text {mic, } \sigma}-r_{\lambda}^{\text {mac }, \sigma}\right)
$$

for $\sigma=2 \mathrm{~km} / \mathrm{s}$ and several values of 1 . Essentially two regions can be distinguished:

1) $0 \leqslant \mathrm{~d}_{\lambda}^{1, \sigma} \leqslant \frac{1}{2}$ : Microturbulence governs the radiative transfer. For $1 \simeq 100 . .500 \mathrm{~km}$ this case applies only to the line cores and the outer wings. We infer that for smaller 1 the entire line is formed under microturbulent conditions.

2) $\frac{1}{2} \leqslant a_{\lambda}^{l, \sigma} \leqslant 1$ : Macroturbulence governs the radiative transfer. This effect is most pronounced at those parts of the profile where the curves of Fig. $1 \mathrm{~b}$ have their maximum. For correlation lengths $1 \gtrsim 1000 \mathrm{~km}$ the entire line is formed under macroturbulent conditions.

Throughout this discussion the UOP has been adopted. Using the KAP would yield quantitatively very similar results. However, due to the underlying cell structure of this model, KAP results always show a higher degree of correlation than the corresponding UOP results obtained for the same line with an identical correlation length and mean square turbulent velocity, (Frisch (1975), Gail et al. (1976)).

3. Empirical Relations between $\left(v_{\text {mic }}, v_{\text {mac }}\right)$ and $(1, \sigma)$

In this section we want to present preliminary results obtained by a cooperation with $\mathrm{H}$. Holweger (Kiel).

There are essentially two reasons which provide the motivation for the 
study of the relations between the classical micro-macroturbulence parameters ( $v_{m i c}$ ' $v_{m a c}$ ) and the fundamental parameters of the mesoturbulence description $(1, \sigma)$.

1) In classical theory $v_{\text {mic }}$ and $v_{\text {mac }}$ have been introduced as free parameters to fit observed and calculated profiles and curves of growth. If it is possible to relate these quantities in a simple way to 1 and $\sigma$ the corresponding parameters of the turbulent velocity field, we arrive at a physically more justified interpretation of $\mathrm{v}_{\mathrm{mic}}$ and $\mathrm{v}_{\mathrm{mac}}$.

2) In the past analyses of stellar atmospheres on the basis of classical micro- macroturbulence theory have been performed providing empirical values for $v_{\text {mic }}$ and $v_{\text {mac }}$. By means of relations between these quantities and $I$ and $\sigma$ one is in the position to determine the mean turbulent velocity and the correlation length of the turbulence field for such atmospheres without reanalizing them by means of the more complex mesoturbulence formalism.

In order to find out the relations between these two sets of parameters the following procedure has been used:

For a given model atmosphere and artificial data for FeI- and FeIIlines curve of growth have been computed for a 1- $\sigma$-grid. On the other hand the conventional microturbulence approach has been used to calculate an independent set of curves of growth for the same transitions. By varying the parameter $v_{\text {mic }}$ an optimum fit between these two sets of data has been found using as criterion the minimum value of

$$
\phi=\sum_{\text {lines }}^{\sum\left[\left(w_{\lambda}^{\text {mic }}-w_{\lambda}^{l, \sigma}\right) / w_{\lambda}^{1, \sigma}\right]^{2} .}
$$

Having thus determined a relation between $v_{\text {mic }}$ and $1, \sigma$ we use the calculated profiles $r_{\Delta \lambda}^{\mathrm{mic}, \mathrm{mac}}$ and $r_{\Delta \lambda}^{1, \sigma}$ to derive an optimum macroturbulent velocity $v_{\text {mac }}$ by minimizing

$$
\psi=\sum_{\text {ines }} \sum_{\Delta \lambda_{j}}\left(r_{\Delta \lambda_{j}}^{\text {mic, mac }}-r_{\left.\Delta \lambda_{j}^{1, \sigma}\right)^{2}}\right. \text {. }
$$

In this way for each pair $(1, \sigma)$ a corresponding pair ( $v_{\text {mic }} v_{\text {mac }}$ ) has been determined empirically.

A typical result for the sun is shown in Fig. 3 where for three different artificial iron lines the optimum values for $v_{m i c}$ and $v_{m a c}$ are plotted versus 1 for a given mean square turbulent velocity $\sigma$. We see the expected strong correlation between the "decrease" of microturbulence and the corresponding "increase" of macroturbulence with growing 
correlation of the turbulence field.

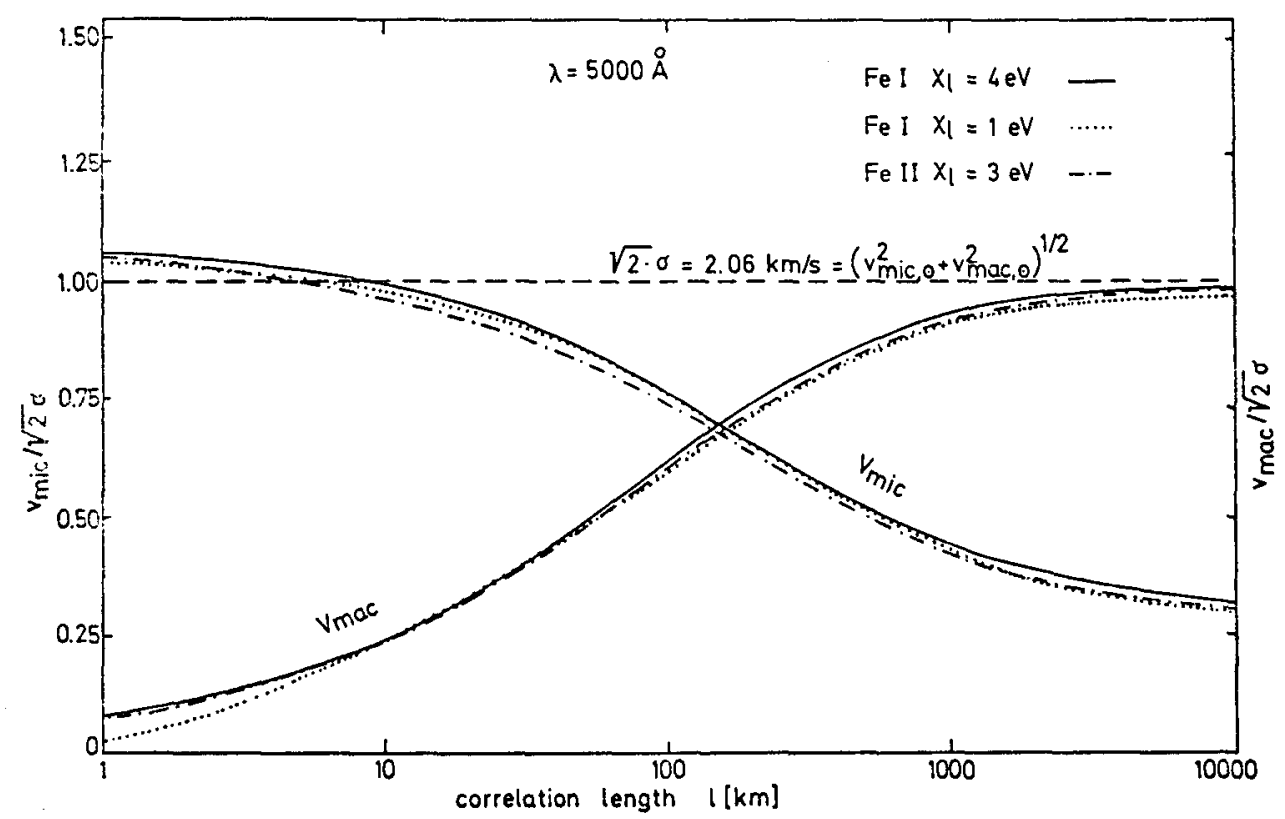

Fig. 3: Empirical dependence of the classical micro- and macroturbulent velocity on the correlation length 1 , derived on the basis of three artificial iron lines with wavelength $\lambda=5000 \mathrm{~A}$ and an excitation energy of the lower level $x_{1}$. (A mean square turbulent velocity $\sqrt{2} \sigma=2.06 \mathrm{~km} / \mathrm{s}$ has been chosen, because this value - according to relation ( 8 ) - corresponds exactly to the empirical values $\mathrm{v}_{\mathrm{mic}, \odot}=1 \mathrm{~km} / \mathrm{s}$ and $\mathrm{v}_{\mathrm{mac}, \odot}=1.8 \mathrm{~km} / \mathrm{s}$. (Empirical model photosphere of Holweger (1967)).

The curves of Fig. 3 resemble to some extent the filter functions introduced by de Jager $(1972,1979)$, de Jager and vermue (1977) and vermue and de Jager (1979) in order to describe the fraction of energy contained in the microturbulent and macroturbulent mode respectively. However, there are significant conceptual differences:

(i) In the description of de Jager and Vermue the adopted definition of microturbulence is based solely on the investigation of weak lines, whereas in our approach the microturbulence velocity is derived by a classical curve of growth analysis with no restriction concerning the line strength.

(ii) The filter function method is based on the consideration of one single mode $u(k)$ of the Fourier spectrum of the turbulent field, whereas our statistical approach by means of probability distributions and correlation functions describes the mean values of the 
turbulent field to which all modes contribute.

The fact, that in the filter function presentation the transition from the micro- to the macroturbulent regime is much steeper than in our approach may be due to this difference.

In order to demonstrate the empirical relations between ( $v_{\text {mic }}$ ' $v_{\text {mac }}$ ) and $(1, \sigma)$ more clearly for an arbitrary line of the solar photosphere the quantity $\left(v_{\text {mic }}^{2}+v_{\text {mac }}^{2}\right)^{1 / 2}$ is plotted in Fig. 4 a versus 1 for a given $\sigma$. This plot provides strong evidence for a relation of the form

$$
2 \sigma^{2}=v_{\mathrm{mic}}^{2}+v_{\mathrm{mac}}^{2}
$$

which seems to hold with considerable accuracy at least within the region of relevant 1 values.

This relation, which has been confirmed by computing a large number of lines, also holds for different stellar atmospheres (AOV, K2III). In no case deviations from the relation (8) of more than few percents have been found.

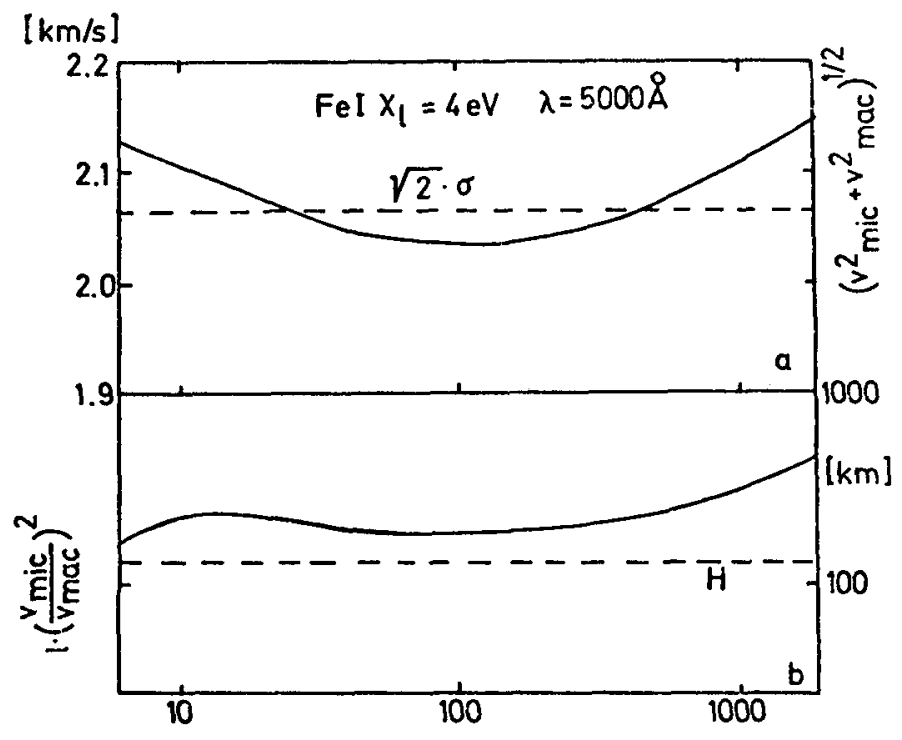

correlation length $1[\mathrm{~km}]$

Fig. 4: The quantities $\left(v_{\text {mic }}^{2}+v_{\text {mac }}^{2}\right)^{1 / 2}$ and $I\left(\frac{v_{\text {mic }}}{v_{\text {mac }}}\right)^{2}$ versus $\lambda$ for the same line as Fig. 3. H is the pressure scale height at the depth of line formation. 
To relate the correlation length 1 to the velocity parameters an additional typical scale length $H$ characterizing the atmosphere has to be introduced. This scale length turns out to be the pressure scale height taken at an optical depth which corresponds to the region of line formation. From Fig. $4 \mathrm{~b}$, where the quantity $l\left(\frac{v_{m i c}}{v_{m a c}}\right)^{2}$ is plotted versus 1 , we conclude that for 1 values which can be mątermined reliably a satisfactory fit is obtained by the relation

$$
\frac{H}{I} \simeq\left(\frac{v_{\text {mic }}}{v_{\text {mac }}}\right)^{2} \text {. }
$$

Relation (9), like (8), has been confirmed for the considered atmospheres by calculating a large number of lines. In no case a deviation of more than 10 percent has been found.

Such empirical relations should reflect similar relations connecting the basic physics of these approaches. In order to give an at least heuristic explanation for these basic relations we require that the corresponding one-point and two-point velocity distribution functions describing classical micro-macroturbulence and mesoturbulence respectively are identical:

$$
\underset{\mathbb{P}_{1}(v)}{\operatorname{mic}, \operatorname{mac}} \equiv \mathbb{P}_{1}^{1, \sigma}(\mathrm{v})
$$

and

$$
\underset{\mathbb{P}_{2}\left(\mathrm{v}_{1}, \mathrm{v}_{2}\right)}{\operatorname{mic}, \operatorname{mac}}=\mathbb{P}_{2}^{l, \sigma}\left(\mathrm{v}_{1}, \mathrm{v}_{2}, \rho\right)
$$

With

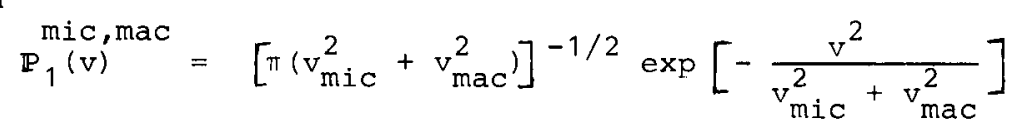

and

$$
\mathbb{P}_{1}^{1, \sigma}(\mathrm{v})=[\pi 2 \sigma]^{-1 / 2} \exp \left[-\frac{\mathrm{v}^{2}}{2 \sigma^{2}}\right]
$$

one immediately obtains relation

(8) $2 \sigma^{2}=v_{\text {mic }}^{2}+v_{\text {mac }}^{2}$. 
From

$$
\begin{aligned}
& \mathbb{P}_{2}\left(v_{1}, v_{2}\right)=\left[\pi^{2} v_{\text {mic }}^{2}\left(v_{\text {mic }}^{2}+2 v_{\text {mac }}^{2}\right)\right]^{-1 / 2} . \\
& \exp \left[-\frac{\left(v_{m i c}^{2}+v_{m a c}^{2}\right)\left(v_{1}^{2}+v_{2}^{2}\right)-2 v_{m a c}^{2} v_{1} v_{2}}{v_{m i c}^{2}\left(v_{m i c}^{2}+2 v_{m a c}^{2}\right)}\right]
\end{aligned}
$$

and

$$
\mathbb{P}_{2}^{l, \sigma}\left(v_{1}, v_{2}, \rho\right)=\left[\pi 2 \sigma^{2}\left(1-\rho^{2}\right)^{1 / 2}\right]^{-1} \exp \left[-\frac{v_{1}^{2}+v_{2}^{2}-2 \rho v_{1} v_{2}}{2 \sigma^{2}\left(1-\rho^{2}\right)}\right]
$$

with

$$
\rho(s)=\exp \left(-\frac{s}{I}\right) .
$$

One sees that eq. (11) can hold only for one particular value of the correlation function $\rho(s)$.

Using eq. (8) one obtains for this particular value

$$
\rho(s)=\frac{v_{\text {mac }}^{2}}{v_{\text {mic }}^{2}+v_{\text {mac }}^{2}}
$$

which for $s \ll 1$ immediately yields the approximate relation

$$
\frac{\mathrm{s}}{1} \simeq\left(\frac{v_{\mathrm{mic}}}{v_{\operatorname{mac}}}\right)^{2}
$$

So at least the analytical form of the empirical result (9) is recovered. As $s$ is a typical geometrical dimension of the line forming region, it should be closely connected with the scale height $H$ introduced in (9).

In order to justify the assumption $s<1$ we plot in Fig. 3 two horizontal lines indicating the empirically determined values for $v_{m i c, \odot}$ and $v_{\text {mac, } \odot}$ respectively (Holweger (1967)). This plot is shown in Fig. $3 a$. The abscissas of the intersection points of each line with the corresponding $\mathrm{v}_{\text {mic }}$ - or $\mathrm{v}_{\text {mac }}$ - curves determine the region of best fitting 1 values. We see that the optimum 1 values are confined to a rather narrow interval of $530 \mathrm{~km} \leqslant 1 \leqslant 640 \mathrm{~km}$. These 1 values are considerably larger than the scale height $\mathrm{H}$ shown in $\mathrm{Fig}$. $4 \mathrm{~b}$, which justifies our above assumption. 


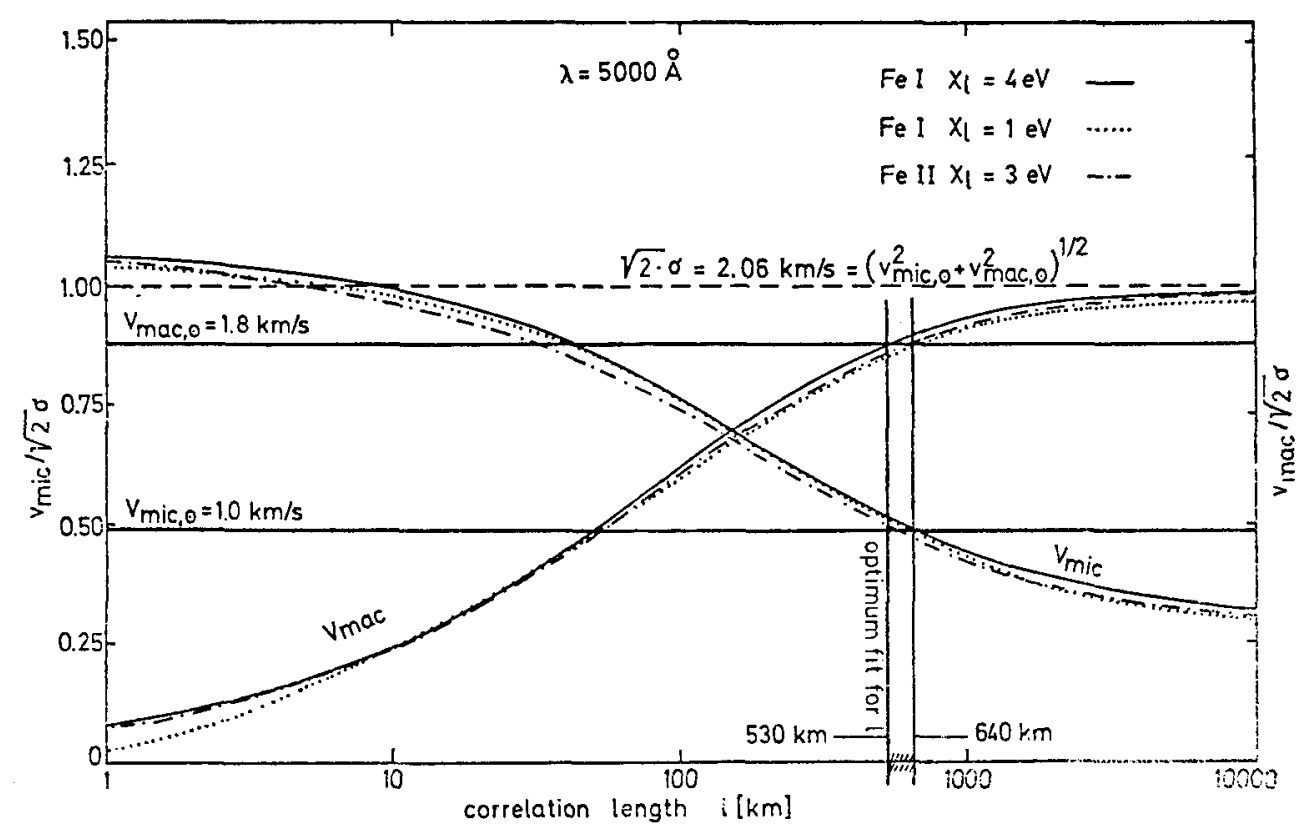

Fig. 3a: See Fig. 3. The horizontal lines indicate the empirically determined photospheric values for $v_{\text {mi }}$ and $v_{\text {mac }}$ (Holweger (1967)). The vertical lines determine the interval of bestefitting l values (strongly crosshatched on the abcissa).

Our result for the optimum correlation of $1 \simeq 600 \mathrm{~km}$ for the solar photosphere is in close agreement with the average value $\frac{2 \pi}{\mathrm{k}} \simeq 600 \mathrm{~km}$ for the inverse wavenumber of the velocity spectrum derived by de Jager and Vermue (1977) by means of the filter function method. The fact that these very different methods yield approximately the same results provides confidence for this value of the scale length.

\section{Conclusion}

Our analysis of the radiative transfer of selected lines in three particular atmospheres (AOV (Wega), G2V (Sun), K2III (Arcturus)) demonstrates that a finite correlation length of the turbulent velocity field exerts a strong influence on the line formation. The general dependence of this effect on the basic parameters $(1, \sigma)$ is similar in all atmospheres and for all profiles considered.

A comparison between profiles and curves of growth computed by means of the mesoturbulence formalism at one hand and by the classical micro- 
macroturbulence method at the other, provides strong evidence for very close relations between $(1, \sigma)$ and $\left(v_{\text {mic }}, v_{\text {mac }}\right)$. These relations allow a reinterpretation of the classical micro-macroturbulence dichotomy which is closer to physics.

Acknowledgements. This work has been performed as part of the program of the Sonderforschungsbereich 132 "Theoretische und praktische Stellarastronomie", which is sponsored by the Deutsche Forschungsgemeinschaft. 


\section{References}

Auvergne, M.; Frisch, H.; Frisch, U.; Froeschlé, Ch.; Pouquet, A.;1973, Astron. \& Astrophys. 29,93

Brissaud, A.; Frisch, U.; 1974, J. Math. Phys., 15, 524

De Jager, C.; 1972, Solar Phys. $\underline{25}, 71$

De Jager, C.; 1978, Astrophys. Space Sci. $\underline{59}, 165$

De Jager, C.; Vermue, J.; 1977, Solar Phys. 54, 313

Frisch, U.; 1968, in Probabilistic Methods in Applied Mathematics, edited by A.T. Bharucha-Reid, Academic Press, New York and London

Frisch, H.; Frisch, U., 1976 in Physique des mouvements dans les atmosphères stellaires, Colloques internationaux du centre national de la recherche scientifique, Paris p.113

Frisch, H.; Frisch, U., 1976, Monthly Notices Roy.Astron. Soc. 175, 157

Gail, H.-P.; Hundt, E.; Kegel, W.H.; Schmid-Burgk, J.; Traving, G., 1974, Astron. \& Astrophys. 32, 65

Gail, H.-P.; Sedlmayr, E., 1974, Astron. \& Astrophys. 36, 17

Gail, H.-P.; Kegel, W.H.; Sedlmayr, E., 1975, Astron. \& Astrophys. $\underline{42,81}$

Gail, H.-P.; Sedlmayr, E.; Traving, G., 1975, Astron. \& Astrophys. $\underline{44,421}$

Gail, H.-P.; Sedlmayr, E.; Traving, G., 1976, Astron. \& Astrophys. $\underline{46,441}$

Gail, H.-P.; Sedlmayr, E.; Traving, G., 1979, JQSRT, in press

Gray, D.F., 1977, Astrophys. J. 218, 530

Gray, D.F., 1978, Solar Phys. $\underline{59}, 193$

Hearn, A.G., 1974, Astron. \& Astrophys. 31, 415

Holweger, H., 1967, Z. Astrophys. $\underline{65}, 365$

Hundt, E., 1973, Astron. \& Astrophys. 29, 17

Magnan, C., 1976, JQSRT 16, 281 
Magnan, C., 1976, in Physique des mouvements dans les atmosphères stellaires, Colloque internationaux du CNRS, Paris p. 179

Rybicki, G., 1976, in Physique des mouvements dans les atmosphères stellaires, Colloque internationaux du CNRS, Paris p. 189

Schmid-Burgk, J., 1974, Astron. \& Astrophys. $\underline{32}, 73$

Sedlmayr, E., 1976, in Physique des mouvements dans les atmosphères stellaires, Colloque internationaux du CNRS, Paris p. 157

Struve, O.; Elvey, C.T., 1934, Astrophys. J. 79, 409

Traving, G., 1964, Z. Astrophys. 60, 167

Traving, G., 1975, in Problems in Stellar Atmospheres and Envelopes eds. B. Baschek, W.H. Kegel and G. Traving, SpringerVerlag, Berlin-Heidelberg-New York p. 325

Traving, G., 1976, in Physique des mouvements dans les atmosphères stellaires, Colloques internationaux du CNRS, Paris p. 145

Vermue, J.; de Jager, C., 1979, Astrophys. Space Sci. 61, 129

Wang, M.CH.; Uhlenbeck, G.E., 1945, Rev. Mod. Phys. 17, 323 